\title{
AN ELECTROMYOGRAPHIC STUDY OF JAW OPENING AND CLOSING REFLEXES IN MAN
}

\author{
Y. Yamada and M. M. Ash JR \\ Stomatognathic Physiology Laboratory, Department of Occlusion, School of Dentistry, \\ University of Michigan, Ann Arbor, MI 48109, U.S.A
}

\begin{abstract}
Summary-The electromyographic activity of the right masseter and digastric muscles was recorded in 10 subjects. A jaw-opening reflex was observed shortly after a mechanical stimulation in subjects performing clenching and active jaw-opening exercises. The latency of this reflex activity was about $28 \mathrm{~ms}$, coinciding with the termination of the silent period of the masseter muscle. The experiments show that this opening reflex can occur without masseter spindle-unloading and may respond to low-threshold afferents.
\end{abstract}

\section{INTRODUCTION}

The control of mastication has been investigated for a long time. Sherrington (1917) suggested that the mechanism of cyclic jaw movement is composed of two main reflexes, i.e. jaw closing and opening, an idea supported by Rioch (1934) who suggested that cyclic jaw movement may be the result of a peripheral controlling mechanism. Although a central control theory for mastication has been accepted generally (Sumi, 1970; Dellow and Lund, 1971), the two reflexes may still be fundamentally separate.

In experimental animals, jaw closing and opening reflexes have been investigated and some neural pathways have been clarified (Sumino, 1971; Matthews, 1975). In man, the jaw-closing reflex has been found to have the same characteristics as in animals; however, although studied by several investigators (Yemm, 1972a,b; Bratzlavsky, De Boever and van der Eecken, 1976; Matthews, 1976), the jaw-opening reflex is not well characterized. In fact, there is still some doubt about its existence.

During ongoing activity of the jaw-closing muscles, a mechanical or electrical stimulus in the facial area results in a brief pause in electromyographic activity which has been reported both in man (Ahlgren, 1969; Yemm, 1972a,b) and in animals (Goldberg, 1972 Gurza, Lowe and Sessle, 1976). In experimental animals, this pause precedes jaw-opening muscle activity (Kawamura and Fujimoto, 1958) in contrast to man, where it does not.

Thexton (1973) proposed that jaw reflexes should be investigated under natural input signals from oral structures. He also suggested that the manifestation of jaw reflexes is affected by experimental conditions (Thexton, 1974). Our aim was to investigate the jaw opening and closing reflexes in man under various jaw conditions with a mechanical stimulation and to clarify some relationships between the jaw opening and closing muscles.

\section{METHODS}

The experiments were performed on 8 males and 2 females with natural dentitions. Ages ranged from 23 to 36 years. No special effort was made to classify the subjects as normal or dysfunctional. However, no subject complained of symptoms of disturbances of the masticatory system. Bipolar recordings were made from the right masseter and right anterior digastric muscles. For the masseter muscle, silver-disc surface clcctrodes were placed approx. $2 \mathrm{~cm}$ apart, centre to centre, in a line parallel to the long axis of the muscle fibres. For the digastric muscle, surface electrodes werc placed approx. $1 \mathrm{~cm}$ apart, close to the insertion of the anterior belly into the mandible, in accordance with Hannam, Matthews and Yemm (1968). In one experiment, a needle clectrode was inserted into the digastric muscle. The ground electrode was placed on the right ear. Subjects performed voluntary movements to confirm that the electrodes werc sampling appropriate signals.

Subjects were seated upright in a dental chair with a conventional head-rest. The recordings of the electromyograms were made as follows:

(1) Tap on the chin while in the rest position; during maximum clenching in the intercuspal (IC) position (i.e. centric occlusion); during maximum clenching in the incisal edge-to-edge contact (IEC) position.

(2) Tap on the chin during active jaw opening; tapping on the labial face of the maxillary right central incisor during active jaw opening.

(3) Rapid tooth-tapping movement in IC position; rapid tooth-tapping movement in IEC position.

The frequencies of stimulation for tapping both the chin and the incisor were approx. 2 per second. The stimuli were always given in groups of 15 with a minimum of $5 \mathrm{~min}$ rest between the last stimulus of one group and the first stimulus of the next. The reflex hammer was fitted with a switch which was closed when the hammer contact with the chin or central incisor and an electrical pulse was delivered which served as the signal for the onset of the stimulus. During rapid tooth-tapping movement, the speed, again 2 per second, was indicated to the subject by a metronome. Tooth contact during rapid tooth-tapping movement was detected by a microphone and for data-processing purposes served the same function as the hammer. 
The electromyographic signals (EMG) were amplified by Grass Model 78 amplifiers with a frequency response approximately linear from $30 \mathrm{~Hz}$ through $3 \mathrm{kHz}$, and taped together with the tap or tooth contact signals (Hewlett-Packard 3955 Instrumentation Recorder). The EMG activity was displayed on a Tektronix $5103 \mathrm{~N}$ storage oscilloscope and samples of 10 superimposed responses were photographed with a Polaroid camera. For small signals, such as digastric muscle activity, a special computer-based data-processing was peifor mied.

After being digitized (sampling rate $175 \mu \mathrm{s} / \mathrm{sample}$ ), the EMG signal was fully rectified and averaged by triggering on the hammer blow or tooth contact. This signal averaging was done on 15 responses from $10 \mathrm{~ms}$ before the trigger to $50 \mathrm{~ms}$ after the trigger. The results werc normalized to the maximum amplitude and plotted by an $x-y$ plotter under computer control.

\section{RESULTS}

\section{Rest position}

By tapping the chin down and backward while the subject's mouth was slightly open and the jaw muscles relaxed, the jaw-jerk reflex was evoked from the masseter muscle (Fig. 1). All subjects exhibited this re- sponse. The latency of the response ranged from 8-10 ms. There was no reflex activity before or after the jaw-jerk reflex in the masseter muscle. In contrast, the signal from the digastric muscle showed great inter- and intra-subject variability. While relaxing, the tap stimulus may displace the mandible and thereby disturb the electrodes (and/or electrode wires), therefore motion artifact should be considered in interpreting the low level activity seen in the digastric muscle recordings. In support of this, we observed that the signals contained primarily low frequencics (Fig. 1A). Thus, the signal from the digastric might have been only motion artifact. The averaged results revealed that the motion artifact appcared at approx. 5-25 ms after the stimulus (Fig. 1B).

\section{Clenching}

The jaw-jerk reflex was also observed during clenching in IC position and IEC position when the stimulus was a chin tap. Subjects were instructed to maintain maximum clenching during these procedures.

Figure 2 shows the reflex activity following chin tap stimulus while the subject was clenching in IC position. The synchronized masseter muscle activity, evident in both positions, could be elicited by tapping on the chin of all the subjects. This reflex activity had a
A

M.

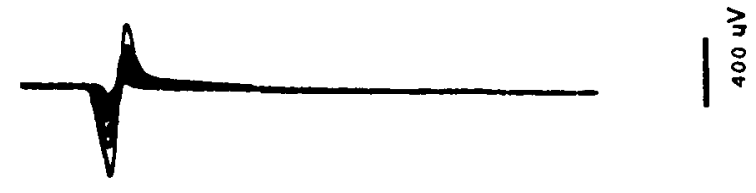

D.
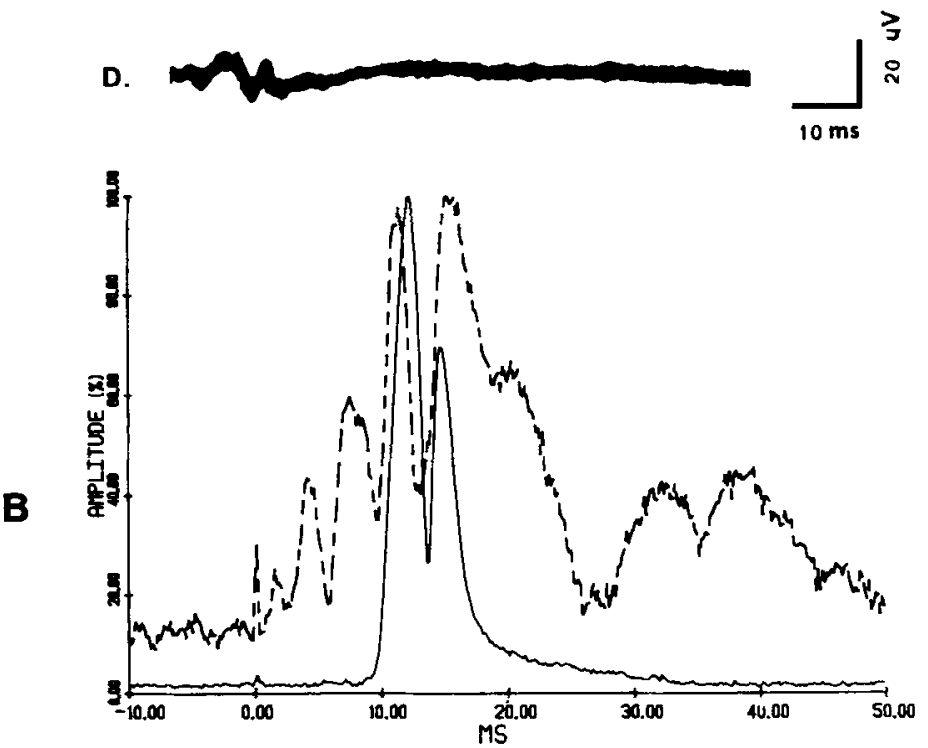

Fig. 1. Reflex activities of the right masseter and digastric muscles. Tap stimuli were delivered on the chin while the subject was relaxing. (A) Ten superimposed oscilloscope sweeps of the two muscle activities. The tap stimulus coincided with the triggering of each sweep. The upper trace indicates the masseter muscle activities and the lower indicates the digastric muscle activities. (B) The average of 15 responscs, with the same activities in (A), from $10 \mathrm{~ms}$ before the stimulus to $50 \mathrm{~ms}$ after the stimulus. The masseter muscle activity indicated by the smooth line and the digastric muscle activity indicated by the broken line were fully rectified before averaging. The stimulus was delivered at the point indicated as $0 \mathrm{~ms}$. Vertical axis is normalized to the maximum average value for each muscle between $10 \mathrm{~ms}$ before the stimulus and $50 \mathrm{~ms}$ after the stimulus. All figures except Fig. 4 were obtained from the same subject. 
M.

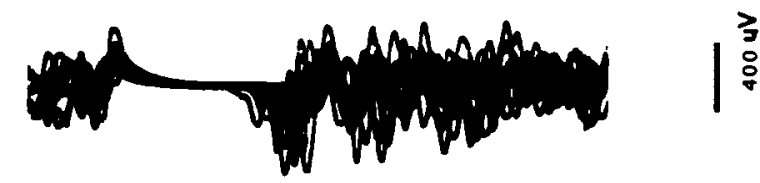

A
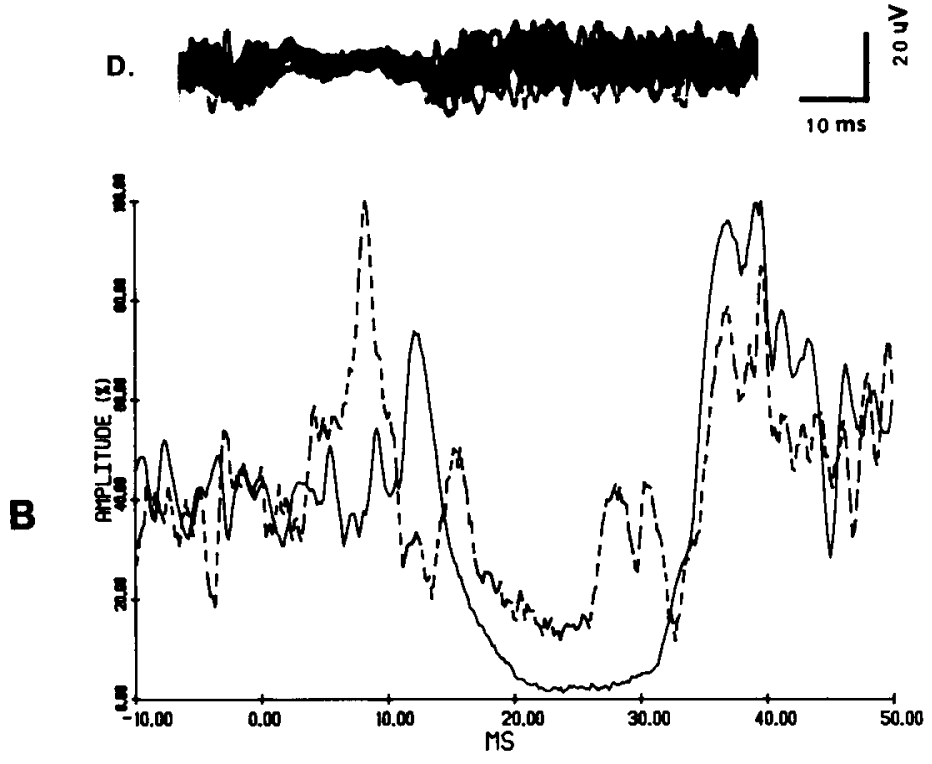

Fig. 2. Reflex activities of the two muscles: tap stimuli were delivered on the chin while the subject maintained maximum clenching in the IC position.

latency of about $10 \mathrm{~ms}$, similar to that observed in rest position. This activity was followed by an inhibitory period and then finally returned to the previous level. The inhibitory period, called the silent period, had a latency of $12 \mathrm{~ms}$ and continued for approx. $20 \mathrm{~ms}$. In 3 subjects, the inhibitory period was followed by a synchronized activity (Fig. 2B).

Similar reflex patterns were seen during clenching in IEC position. Although masseter muscle activity was lower than that during clenching in IC (Fig. 3A), the averaged results, which are normalized, (Fig. 3B) indicated that there was no difference in the reflex patterns.

Although the masseter muscle produced the expected activities, the digastric muscle showed activity not previously reported. In 6 of the 10 subjects, synchronized digastric muscle activity was observed shortly after the tap stimulus while clenching in IC position. As the digastric muscle was not very active during clenching in IC position, the averaged results exhibit much more information than the raw data. In Fig. 2B, the digastric muscle shows four peaks, the first two at 9 and $16 \mathrm{~ms}$, the next one at approx. $28 \mathrm{~ms}$ and the last one at $35 \mathrm{~ms}$ after the stimulation. The peaks at 9 and $16 \mathrm{~ms}$ may be the motion artifact previously mentioned. Other subjects had almost the same peaks (Fig. 4). In Fig. 4A, the synchronized digastric muscle activity is clear even in the raw data. The activity at $28 \mathrm{~ms}$ appeared consistently whereas activity at $35-45 \mathrm{~ms}$ was sometimes less pronounced.

Clenching in IEC position produced background activity in the digastric muscle in most subjects, although the amplitude was small. In three cases, the digastric reflex activity was more clear in IEC than in IC position (Fig. 3). In some cases, there was a silent period of the digastric muscle.

\section{Active opening}

In 9 of the 10 subjects, a jaw-opening reflex of the digastric muscle was observed during active jaw opening. For this experiment, the subjects were instructed to sustain a moderate opening effort of their jaw against their thumb placed under the chin. The tap stimuli were delivered on the chin and on the maxillary right central incisor during active jaw opening. In three cases, two opening efforts were tested. These can he considered maximal and moderate.

During active jaw opening, the tap stimuli were delivered on the chin and on the maxillary right central incisor. Although the masseter muscle reflex activity was observed only with a tap stimulus on the chin, the digastric muscle activity was observed with both stimuli. The peak of this digastric reflex activity coincided with the peak observed during clenching (Figs 5 and 6).

When the tap stimulus was delivered to the chin, the masseter muscle activity with a latency of approx. $10 \mathrm{~ms}$ was temporally the same as that observed for the same stimulus while the jaw muscles were relaxed; i.e. the jaw-jerk reflex. However, with the tap stimulus on the incisor tooth, neither raw data nor averaged result showed any reflex activity in the masseter muscle (Figs 5 and 6).

Both stimuli could elicit the same jaw-opening reflex in the digastric muscle at approx. $28 \mathrm{~ms}$ and $35-45 \mathrm{~ms}$ after the stimulus. The first peak was ob- 
M.

\begin{tabular}{l}
3 \\
0 \\
0 \\
\hdashline
\end{tabular}

A
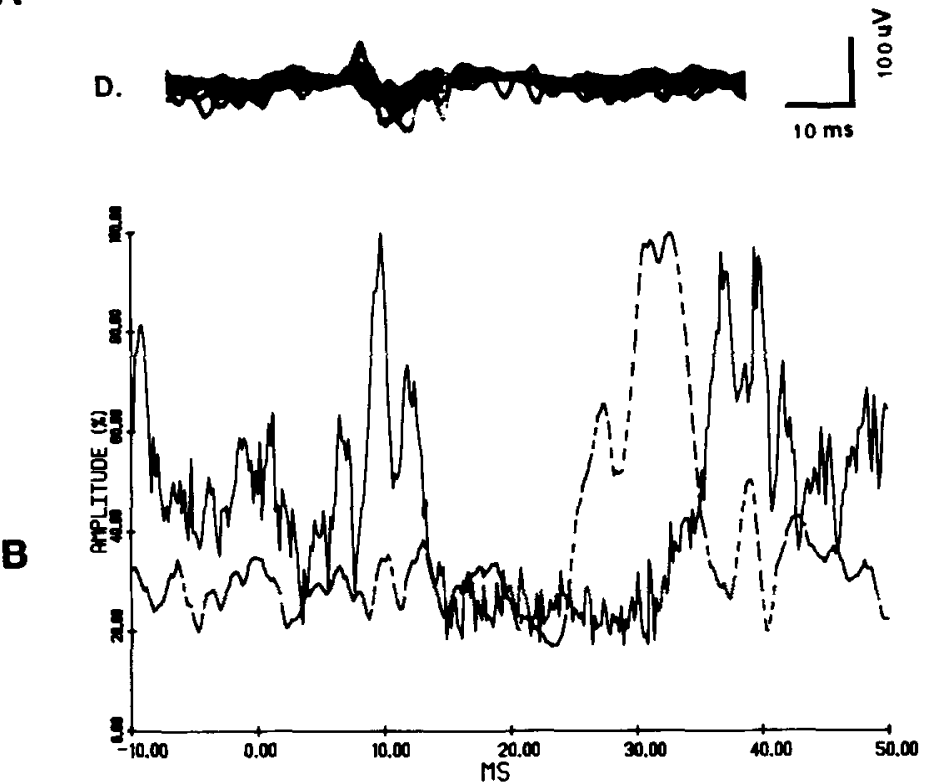

Fig. 3. Reflex activities of the muscles: tap stimuli were delivered on the chin while the subject maintained maximum clenching in the IEC position.

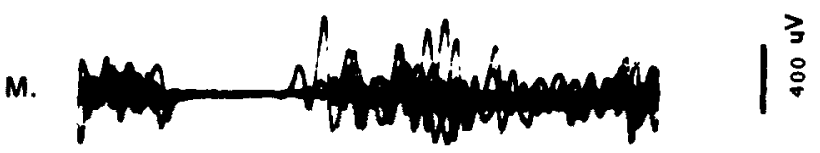

A
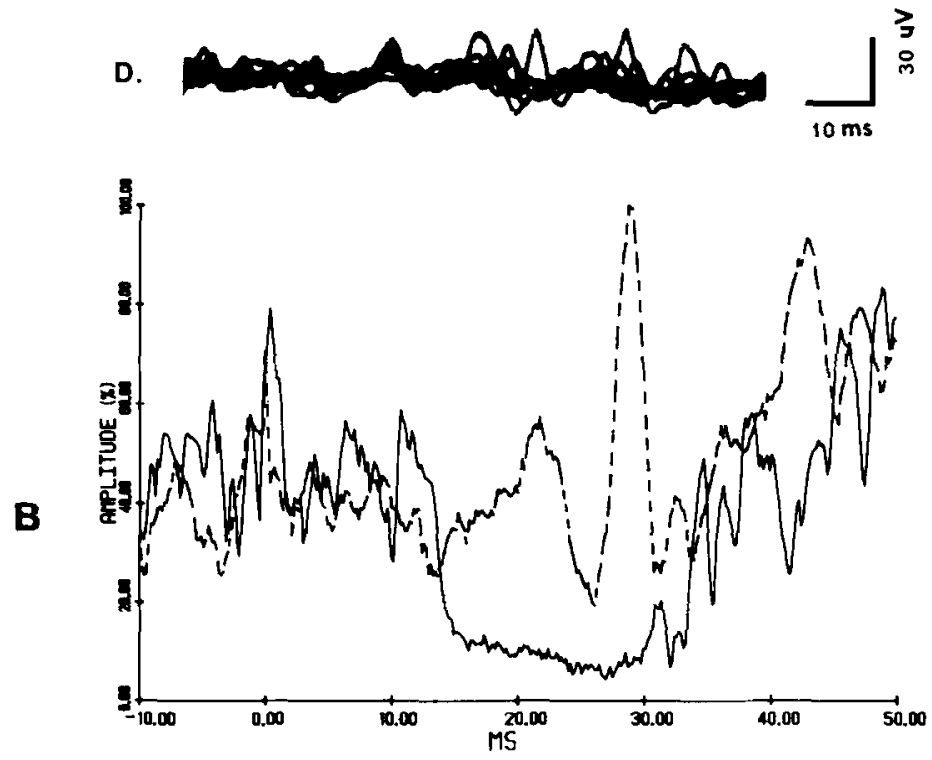

Fig. 4. Reflex activities of the two muscles from another subject: tap stimuli were delivered on the chin while the subject maintained maximum clenching in the IC position. 

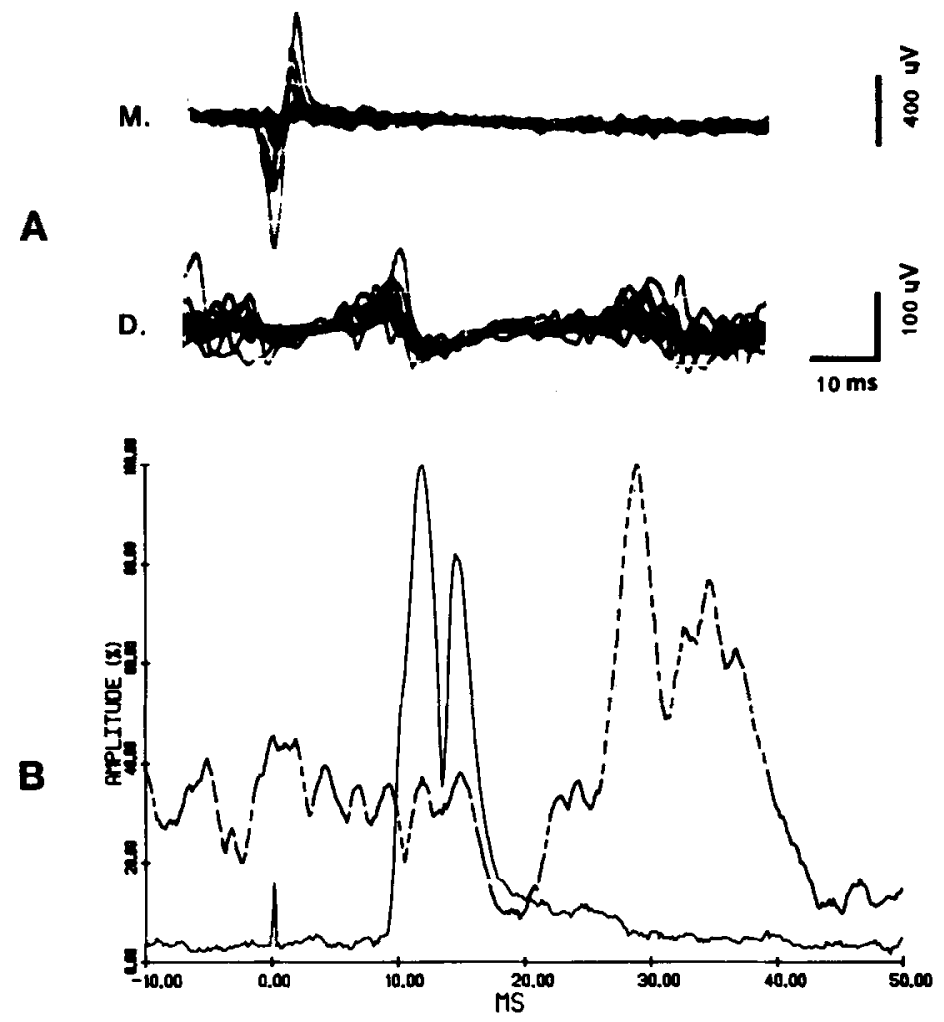

Fig. 5. Reflex activities of the two muscles: tap stimuli were delivered on the chin while the subject maintained moderate jaw opening effort.

M. 亭

A
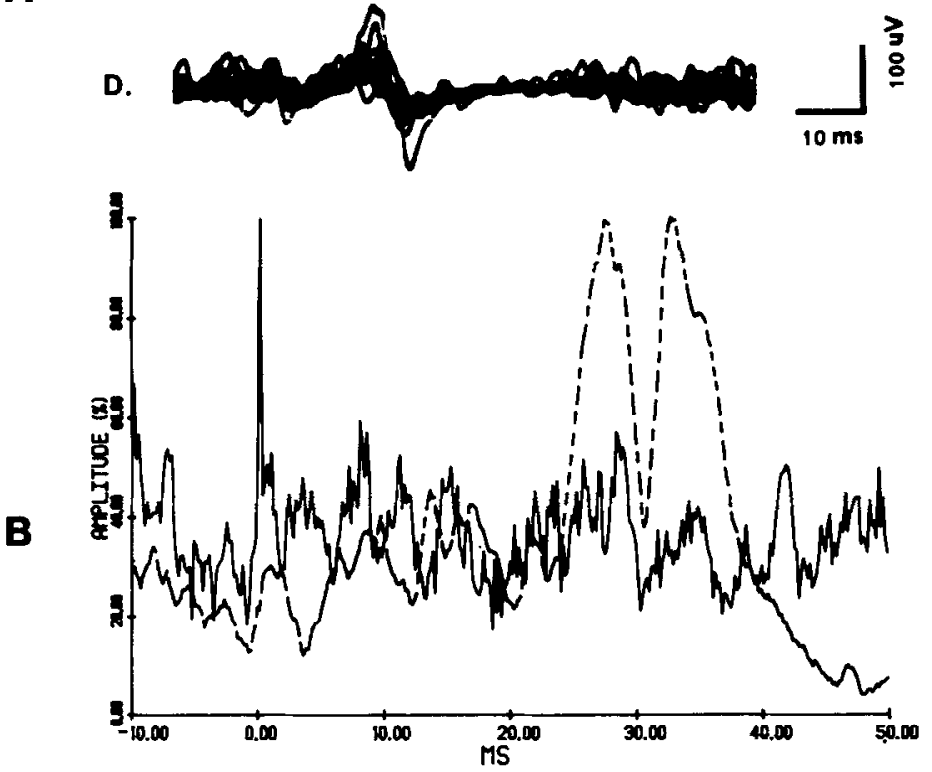

Fig. 6. Reflex activities of the two muscles: tap stimuli were delivered on the maxillary right central incisor while the subject maintained moderate jaw-opening effort. 
A

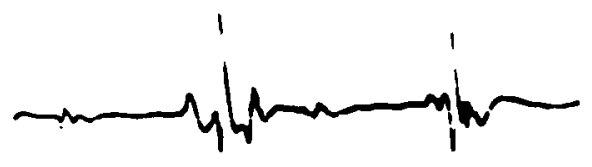

B

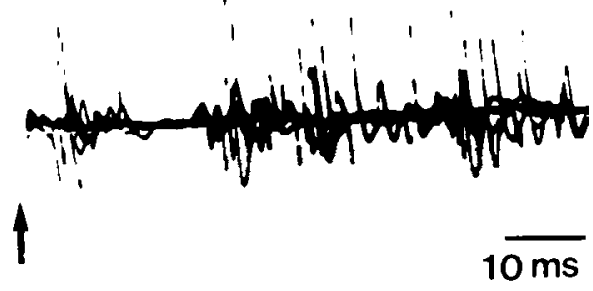

Fig. 7. Digastric activity measured by the needle electrode. The tap stimuli were delivered on the labial face of the maxillary right central incisor while the subject maintained moderate jaw-opening effort. The tap stimulus coincided with the triggering of each sweep. (A) A typical unit activity. (B) Five superimposed oscilloscope sweeps.

served more consistently than the second. Moderate background activity in the digastric muscle emphasized the jaw-opening reflex. However, strong background activity in the digastric muscle masked the jaw-opening reflex so that the raw data did not show a clear jaw-opening reflex.

During active jaw opening, in most subjects, the silent period of the digastric muscle was observed. The latency was about $10-13 \mathrm{~ms}$ and the duration was approx. $10 \mathrm{~ms}$.

To explore the questions of motion artifact and contamination of the digastric surface EMG record by nearby muscles, a needle electrode was inserted into the right digastric muscle and recordings made during active jaw opening. Tap stimuli were delivered on the labial face of the maxillary right central incisor. The synchronized activities were elicited at about 26 and $35 \mathrm{~ms}$ after the stimulus (Fig. 7B). Figure 7 shows a typical unit activity.

\section{Rapid tooth-tapping movement}

During rapid tooth-tapping movement, there was synchronized activity in the digastric muscle at the same latency. However, because of the large motion artifact associated with such vigorous movement, it is difficult to say whether this activity was the jaw-opening reflex.

\section{DISCUSSION}

In our experiments, electrical activities were recorded from the right masseter and the right digastric muscles while subjects maintained background activity in the masseter muscle or in the digastric muscle. During these procedures, a tap stimulus on the chin and right maxillary incisor resulted in jawopening and/or-closing reflexes. Although the masseter muscle produced expected reflexes, the digastric muscle showed activity which conflicts with previous reports.

Because the digastric muscle activity was very weak and tended to be contaminated with motion artifact from the tap stimulus, rest position data were used to characterize the artifact. The averaged results revealed that the motion artifact appeared $5-20 \mathrm{~ms}$ after the stimulus. The nature of this artifact was modulated by the jaw conditions. Because the jaw is more stable during clenches than in rest position, the motion artifact is smaller. During active opening, the digastric muscle activity was large relative to the motion artifact. The unit activities, measured by the needle electrode, concur with the results from the surface electrode and support the assumption that the reflex activities of the digastric muscle are not contaminated by the motion artifact nor the activity from other muscles. During rapid tooth-tapping movement, the artifact was so large that it was not possible to separate the reflex activity from the motion artifact.

\section{Jaw-opening reflex}

In experimental animals, afferents from the oral rcceptors elicit the digastric jaw-oping reflex both in rest position and while clenching. In man, however, Yemm (1972a) reported that the afferents from oral receptors elicit only passive jaw opening during clenching, i.e. the short period of inhibition of the jaw-closing muscles. The active opening reflex has not been observed in rest position, during clenching or during active opening (Yemm, 1972b; Matthews, 1975; Gilling and Klineberg, 1975).

Our experiments employed natural stimulation under various jaw conditions. A tap stimulus delivered on the chin or maxillary incisor elicited the digastric jaw-opening reflex about $28 \mathrm{~ms}$ after the tap. This latency is consistent with those of the unloading reflex of Hannam et al. (1968) and the acoustic jaw reflex of Meier-Ewert, Gleitsmann and Reiter (1974). Hannam et al. (1968) suggested that unloading of the closing muscle spindle may be one of the factors resulting in the reflex activity of the depressor muscle. However, the jaw-opening reflex we observed cannot be explained by this mechanism. Our jaw-opening reflex was elicited by a tap stimulus on the chin and on the maxillary incisor, whereas the jaw-jerk reflex was elicited only by tapping on the chin. These findings suggest that the jaw-opening reflex can occur without masseter spindle unloading. Furthermore, acoustic stimuli, which elicit the same reflex activity in the jaw depressor, could not result in unloading of the muscle spindle. Thus, natural stimulation such as mechanical, unloading or acoustic stimuli may elicit the active jaw-opening reflex.

According to Matthews (1975), there is no digastric jaw-opening reflex in man. His experiments used both electrical and mechanical stimulation under resting, clenching, and active-opening jaw conditions. However, his data analysis did not include an averaging technique. Furthermore, we found that the jaw-opening reflex could not be elicited as easily during a clench in IC position as during active jaw opening with moderate effort. Thus, the digastric jaw-opening reflex seems to be emphasized by background activity in the digastric muscle. Thus, the clenching procedure used by Matthews might also explain the failure to elicit a clear digastric jaw-opening reflex.

The nature of the stimulation also seems to be important for the digastric reflex activity. The afferent, which is involved in the reflex, seems to have a low 
threshold. It is possible that high threshold receptors, when stimulated simultaneously, might mask the digastric jaw-opening reflex. Yemm (1972a) found no digastric muscle activity when electrical stimulation was used in conjunction with an averaging technique. Mat thews (1975), also reported that a tap stimulus on the teeth caused the silent period of the digastric muscle, whereas, electrical stimulation could not elicit the silent period.

The total evidence suggests the following conclusions: (1) The digastric muscle reflex tends to respond to low threshold afferents. (2) Noxious stimulation may mask or inhibit the reflex. (3) Moderate background activity in the depressor muscle may be essential for the reflex.

\section{Jaw-closing reflex}

The jaw-jerk reflex of the masseter muscle was observed in varying degrees in all the procedures employing chin-tap stimulation. For rest position and active opening recordings, the latency of this reflex was longer than the latency observed for clenching. These results concur with those of McIntyre and Robinson (1959) and Goldberg (1972).

Masseter muscle activity similar to the jaw-jerk elicited by stimulation of periodontal receptors during a clench, has been reported as the periodontal masseteric reflex by Goldberg (1971). Our results, however, show that during active opening a tap stimulus on the maxillary incisor fails to elicit any reflex activity in the masseter muscle (Fig. 6). Based upon his report, the mechanism for the periodontal masseteric reflex is an electrical coupling of the cell bodies of primary afferent fibres from periodontal and gingival receptors and afferents from muscle spindles of the jaw closing muscle. As in our results the tap stimulus on the maxillary incisor elicited reflex changes of the digastric muscle activity, we estimate the stimulus to be sufficient to elicit the afferent firing of the periodontal receptors. Therefore, we suggest that the active opening effort may mask this periodontal reflex or, as suggested by Matthews (1975) a mechanism other than electrical coupling of the cell bodies of the primary afferents should be considered for his periodontal masseteric reflex.

\section{Reciprocity of the two muscles}

The digastric activity at approx. $28 \mathrm{~ms}$ coincides with the termination of the silent period of the masseter muscle. Thus, the two muscles seem to show reciprocity. However, the digastric muscle activity at 35-45 ms was not always associated with inhibited masseter muscle activity.

A potentially diagnostic value for the silent period of the jaw-closing muscle has been considered (Bessett, Bishop and Mahl, 1971; Bailey, 1976). Although there still is a question regarding this application (Yemm, 1976) the simultaneous recording of the masseter and the digastric muscle activities might contribute to a resolution of this problem.

Acknowledgements-We wish to thank $\mathrm{Mr}$ Patrick $\mathrm{M}$ Steusloff for his assistance. This investigation was supported by USPHS Grant 02731-21.

\section{REFERENCES}

Ahlgren J. 1969. The silent period in the EMG of the jaw muscles during mastication and its relationship to tooth contact. Acta odont. scand. 27, 219-227.

Bailey J. O. Jr 1976. I he electromyographic silent period in normal subjects and patients with TMJ muscle pain dysfunction. M.S. Thesis, The University of Michigan, School of Dentistry, Ann Arbor, Mich., U.S.A

Bessette R., Bishop B. and Mohl N. D. 1971. Duration of masseteric silent period in patient with TMJ syndrome. J. appl. Physiol. 30, 864-869.

Bratzlavsky M., De Boever J. and van der Eecken H. 1976. Tooth pulpal reflexes in jaw musculature in man. Archs oral Biol. 21, 491-493.

Dellow P. G. and Lund J. P. 1971. Evidence for central timing of rhythmical mastication. J. Physiol., Lond. 215, 1-13.

Gilling B. R. D. and Klineberg I. J. 1975. Latency and inhibition of human masticatory muscles following stimuli. J. dent. Res. 54, 269-279.

Goldberg L. J. 1971. Masseter muscle excitation induced by stimulation of periodontal and gingival receptors in man. Brain Res. 32, 369-381.

Goldberg L. J. 1972. An excitatory component of the jaw opening reflex in the temporal and masseter muscles of cats and monkeys. Experientia 28, 44-46.

Gurza S., Lowe A. A. and Sessle B. J. 1976. Influences on masseter activity of stimuli applied to various sites in cats and macaque monkeys. Archs oral Biol. 21, 705-707.

Hannam A. G., Matthews B. and Yemm R. 1968. The unloading reflex in masticatory muscle of man. Archs oral Biol. 13, 361-364.

Kawamura Y. and Fujimoto J. 1958. A study on the jaw opening reflex. Med. J. Osaka Univ. 9, 377-386.

Matthews B. 1975. Mastication. In: Applied Physiology of the Mouth (Edited by Lavelle C.) Chap. 10. Wright, Bristol.

Matthews B. 1976. Reflexes elicitable from the jaw muscles in man. In: Mastication (Edited by Anderson D. J. and Matthews B.) Chap. 17. Wright, Bristol.

McIntyre A. K. and Robinson R. K. 1959. Pathway for the jaw jerk in man. Brain 82, 468-474.

Meier-Ewert K., Gleitsmann K. and Reiter F. 1974. Acoustic jaw reflex in man: its relationship to other brain-stem and microreflexes. Electromyog. clin. Neurophysiol. 36, 629637

Rioch J. M. 1934. The neural mechanism of mastication Am. J. Physiol. 108, 168-176.

Sherrington C. S. 1917. Reflexes elicitable in the cat from pinna vibrissae and jaws. J. Physiol., Lond. 51, 404-431.

Sumi T. 1970. Activity in single hypoglossal fibers during cortically-induced swallowing and chewing in rabbits. Pflügers Arch. ges. Physiol. 314, 329-346.

Sumino R. 1971. Central neural pathways involved in jawopening reflex in the cat. In: Oral Facial Sensory and Motor Mechanisms (Edited by Dubner R. and Kawamura Y.) pp. 315-332.

Thexton A. J. 1973. Oral reflexes elicited by mechanical stimulation of palatal mucosa in the cat. Archs oral Biol. 18, 971-980.

Thexton A. J. 1974. Jaw opening and jaw closing reflexes in the cat. Brain Res. 66, 425-433.

Yemm R. 1972a. The response of the masseter and temporal muscles following electrical stimulation of oral mucous membrane in man. Archs oral Biol. 17, 23-33.

Yemm R. 1972b. Reflex jaw opening following electrical stimulation of oral mucous membrane in man. Archs oral Biol. 17, 513-523.

Yemm R. 1976. Neurophysiologic studies of temporomandibular joint dysfunction. Oral Sci. Rev. 7, 31-53. 